\title{
Discrete Versus Continual Description of Solid State Reaction Dynamics from the Angle of Meaningful Simulation
}

\author{
A. KOROBOV \\ Kharkov University, P.O.Box 10313, Kharkov 310023, Ukraine
}

(Received 11 March 1998)

\begin{abstract}
Chemical dynamics provides quite a number of examples of interesting and useful discrete models. But it catches one's eye that the majority of them are from the field of homogeneous chemistry. Whereas the chemical individuality of solid substances is represented in discrete terms of crystal lattices, the conventional description of solid state reaction dynamics is essentially continual. The recent progress in the theory of random mosaics and theory of planigons opens the way for developing an alternative discrete description in terms of Dirichlet tessellations. In the present paper the two approaches are compared from the angle of meaningful simulation. It seems that this may be of interest not only for chemists but also in the broad context of developing and employing discrete dynamical models.
\end{abstract}

Keywords: Solid state reactions, Discrete dynamics, Geometric-probabilistic phenomenology, Dirichlet tessellations, Cellular automata

\section{INTRODUCTION}

Both discrete and continual features are generally inherent for real phenomena and processes and may be exhibited in a larger or smaller measure depending on a particular angle they are considered from.

Many interesting examples of this may be found in various branches of science. Aiming to discuss this point with respect to the dynamics of solid state chemical reactions, refer the series of Shubnikov's (1975) works on crystal symmetry. They provide convincing evidence that the coexistence of such seemingly contradictory and mutually exclusive features as continuity and discontinuity within the same crystal are not simply possible but logically necessary. Thus, one and the same crystal may possess, say, the cubic symmetry with respect to some properties and at the same time the spherical symmetry with respect to other properties, exhibiting discrete and continual features respectively.

Upon transition to a mathematical description, the picture becomes distinctly continual or discrete, i.e. this transition involves an important choice.

At this point we face a thought-provoking perplexity concerning the mathematical description of solid state reaction dynamics. The chemical features of crystals are described in discrete terms of crystal lattices. From the angle of heterogeneous 
chemical kinetics such crystals are solid reagents, i.e. the medium in which one or another solid state reaction proceeds. It should seem that discrete space and time naturally suggest themselves in this case for describing the reaction dynamics. But the conventional geometric-probabilistic description of these reactions, originated in the classical works of Kolmogorov (1937), Johnson and Mehl (1939), and Avrami (1941), is essentially continual (modern presentation may be found, in particular, in Barret, 1973; Belen'kiy, 1980; Brown et al., 1980; Delmon, 1969; Sestak, 1984). This is even more strange with the account that in the contiguous field of homogeneous chemical kinetics, where the introduction of discrete variables is not so direct, the discrete ideology has been finding much wider use (see Kapral, 1991 for review).

Figure 1 gives an idea about the situation. The conventional continual formalism was developed for describing the experimentally observed "ratetime" dependencies. Now it is clear that they are determined by universal geometrical regularities of first-order phase transitions conjugated with solid state reactions. The continual approach is efficient for describing these regularities but only until one attempts to get a keener insight into the chemical mechanism of a reaction. Then a number of essential difficulties arise connected with the fact that the main variables has the geometric-probabilistic rather than chemical meaning, i.e. the chemical individuality of a solid reagent is not represented within the continual formalism in the proper manner.

On the other hand, this chemical individuality may be naturally described in discrete terms determined by the crystal chemical structure. But for discussing in these terms the observed macroscopic behaviour the geometry of the crystal space must be agreed with the geometry of the evolution of the reaction front with the account of relevant probabilistic aspects. A possibility to do this is connected with relatively recent progress in the theory of random tessellations (see Møller, 1992; 1995, Stoyan et al., 1987, Chiu, 1995 and references therein) and theory of planigons developed in Delone et al. (1978) and Grünbaum and Shephard (1987). This opens the way for developing a discrete description of solid state reaction dynamics with the primary aim to get a keener insight into interrelations "Chemical individuality $\leftrightarrow$ Mechanism $\leftrightarrow$ Rate. (In Bradley (1987) and Weinberg and Kapral (1989) the lattice analog of Johnson-MehlAvrami equation is considered irrespectively of relevant chemical aspects.)

It should be noted that "from-the-very-beginning" discrete description is implied in the present context (rather than the substitution of a discrete model for a continual one as is often the case when net methods are used on the stage of computations). Mathematics is not simply a language but the language with the logic, the tool for considerations (Feynman, 1965). Accordingly, the change of the

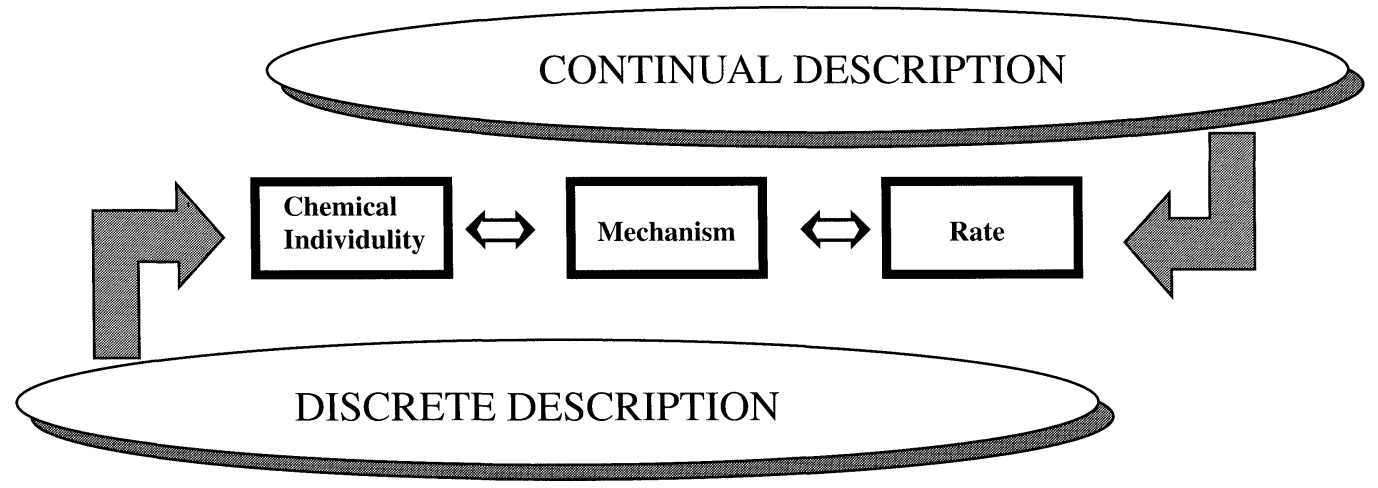

FIGURE 1 In describing the dynamics of solid state chemical reactions the problem is approached in discrete and continual terms from the opposite sides. 
formalism means the change of the "conceptual capability". It seems that now the further accumulation of experimental data is not so crucial for solving the essential issues of solid state reaction dynamics as the progress in their interpretation. In balancing the experimental evidence and the logical substantiation (the external justification and internal perfection according to Einstein) the stress is shifted towards the latter.

\section{A BRIEF OUTLINE AND SOME PECULIARITIES OF THE CONVENTIONAL CONTINUAL DESCRIPTION}

The continual geometric-probabilistic approach to solid state reaction dynamics was discussed from various angles in quite a number of treatises and papers (see, in particular, Barret 1973; Belen'kiy, 1980; Brown et al., 1980; Delmon, 1969; Šestak, 1984). Here we will restrict ourselves to its brief outline and discussion of some particular features.

Crucial premises: The preferred occurrence of a solid state reaction and one and the same sigmoid form of the 'degree of conversion $\alpha$ - time $t$ ' curves for fairly disparate in nature reactions. Main principles: A reaction is localized at the boundary between original and newly formed phases; it proceeds through the formation and growth of new phase nuclei; these nuclei appear in a random manner at the points of energetic inhomogeneity of original phase; the impingement of two nuclei stops their growth in this direction. Main quantities: Time $t$, the degree of conversion $\alpha(t)$, the rate $\alpha(t)$, the kinetic coefficient $k$. Some derived quantities: The nucleation law $L_{\mathrm{n}}(t)$ is the intensity of nuclei formation, the nucleus growth law $L_{\mathrm{g}}(\tau, t)$ is a measure (volume or area) of a growing nucleus; the form factor $\eta$, the age of a nucleus $(t-\tau)$ (where $\tau$ is the instance of nucleus appearance). The central problem in developing the mathematical description: The account of the impingements of growing nuclei. The way of solving it: The degree of conversion $\alpha$ is identified with some probability $P$ that an arbitrary point of the original phase will have appeared within a new phase up to the instant $t$. Main assumptions: The original phase is unrestricted; the nucleation is according to Poisson; the form and orientation of all growing convex nuclei are the same; the rate of a nucleus growth is independent of its age; these assumptions cannot be relaxed (Belen'kiy, 1980). The main model:

$$
\alpha(t) \equiv P=1-\exp \left(-\int_{0}^{t} L_{\mathrm{n}}(\tau) L_{\mathrm{g}}(\tau, t) \mathrm{d} \tau\right) .
$$

The relationship for the rate:

$$
\begin{aligned}
\dot{\alpha}(t)= & w(t) \cdot 2 k \int_{0}^{t}\left[L_{\mathrm{n}}(\tau) \int_{\tau}^{t} w(\xi) \mathrm{d} \xi\right] \mathrm{d} \tau \\
& \cdot \exp \left(-\int_{0}^{t} L_{\mathrm{n}}(\tau) L_{\mathrm{g}}(\tau, t) \mathrm{d} \tau\right)
\end{aligned}
$$

where $w$ is the rate of a separate nucleus growth, and $\xi$ is the integration variable. Some important characteristic features: Mathematical models are independent on the dimensionality; the spherical form of nuclei is chosen as a rule to satisfy one of the mentioned assumptions; the direct proportionality between the rate and the total length of the interface follows from the mathematical formalism without any additional assumptions. Crucial disadvantages: Though rigorous with respect to the nuclei impingement simulation, the geometric-probabilistic formalism is essentially ambiguous when the inverse kinetic problem (IKP) is concerned, resulting in the numerous failures in experimental data interpretation (Korobov, 1994).

Attempts to get at the roots of this ambiguity make explicit some peculiar features of the conventional description relevant in comparing continual and discrete approaches.

In contrast to chemical thermodynamics, which is the direct logical extension of physical thermodynamics, chemical kinetics has been developing from the very beginning in its own ways, fairly disparate for homogeneous and heterogeneous kinetics. As a result, solid state reaction dynamics is characterized by its particular manner of reasoning 
and mathematical formalism. Rather dissimilar from the formalism of homogeneous chemical kinetics, this phenomenology has its main roots in the theory of first-order phase transitions. With the account that solid state reactions are always accompanied by first-order phase transitions, this phenomenology is efficient for describing the universal geometrical regularities of solid state reactions that determine the observed kinetic behaviour i.e. the always sigmoid form of rate-time curves. But the other coin of this universality is that no definite boundary may be drawn between first-order phase transitions proceeding with and without chemical transformations. Gradually the evidence was accumulated that one and the same set of experimental data may be described equally well within this phenomenology by several different mathematical models (see, for example, Brown et al., 1980; Delmon, 1969). This emphasizes, in particular, the need of a deeper insight into the interrelations between observed kinetic behaviour and mechanism. In this connection we arrive at the question to what extent the conventional approach is adequate for this.

In answering this question the geometric-probabilistic rather than chemical meaning of the main variables must be noted, due to which all conceptual details of one or another mechanism that do not fall within this interpretation are represented on the stage of formalization by the model coefficients alone, i.e. they participate in the discrimination procedure not through the form of the model function but only through the numerical values of the coefficients. One of the negative results is that fairly different original concepts concerning the reaction mechanism may lead to the same equation in terms of $\alpha$; examples may be found, in particular, in Delmon (1969) and Prodan (1990).

The conventional phenomenology of solid state reaction dynamics has been using in heterogeneous kinetics for 60 years. During this period the philosophy of phenomenological description has been modifying materially, mainly due to the recent progress in synergetics and complexity theory (Haken, 1983; Nicolis and Prigogine, 1989). In the context of "eliminating the extra variables" (Haken, 1983; Careri, 1982) we realize how tremendous the distance between micro and macro is, in the case of solid state reactions. It becomes clear that complex heterogeneous systems possess phenomenological features that may hardly be discerned in considering more simple subsystems or through direct extrapolation of microscopic properties. One of the main conclusions is that one or another formalization of the mechanism must be based on an adequate phenomenological character representation of the chemical individuality of a solid reagent. In terms of the conventional continual approach the solid reagent is represented as a "sample" consisted of some abstract (as a rule spherical) "particles". This is obviously not sufficient for the close discussion of mechanisms of solid state reactions, and the potential of the continual description seems to be limited in this respect.

\section{DISCRETE DESCRIPTION IN TERMS OF DIRICHLET TESSELLATIONS}

Classical works of Kolmogorov (1937) and Johnson and Mehl (1939) gave rise to the conventional continual approach discussed above. Historically these works played also an important role in developing the theory of random tessellations. Relatively recent progress in this field of contemporary stochastic geometry makes it possible to approach some essential issues of solid state reaction kinetics from a different angle. The use of the language of random tessellations in combination with the language of planigon tessellations opens the way for developing a from-the-verybeginning discrete description of solid state reaction kinetics.

\subsection{Discrete Space: From a Polycrystalline Sample to the Single Crystal Face as a Chemical Individual}

Theoretical description of solid state reaction kinetics was initially oriented to experimental 
results for polydisperse polycrystalline samples characterized by "prehistory" (in what way the sample is prepared, its age, storage conditions, etc.). For quite a long period of time the vast majority of experimental works was of this kind. But during last decades the situation is considerably changed: the fraction of experiments devoted (at least partly) to single crystals has been considerably increasing. Theory does not keep pace with this tendency. The conventional continual approach discussed above is not capable of distinguishing single crystals and polycrystalline samples because in its framework there are no adequate means for representing a solid reagent in terms of its composition and crystal chemical structure. As a result, the same equations are used in both cases.

In this context, a discrete space appears as a result of attempts to overcome this chemical facelessness of the conventional formalism.

It was mentioned that a solid state chemical reaction is always accompanied by first-order phase transitions, and historically the continual geometric-probabilistic approach originated from the theory of first-order phase transitions. But the emphasis is on the formation of new phases alone, the disappearance of the original crystal phase being practically ignored. Until one deals with chemical transformations of polydisperse polycrystalline reagents treated as "samples" (Fig. 2(a)) into polydisperse polycrystalline products, this remains in the shadow and is masked to a degree by the use of formal adjustment parameters. But the picture is changed when a single crystal is taken as an original solid reagent.

Further a single crystal will be considered as a medium in which a solid state reaction proceeds and as a solid reagent simultaneously. Accordingly, this will be a finite real single crystal with defects restricted by crystal faces rather than ideal infinite crystal structure. In the present context it is reasonable to treat such a crystal as a set of growth pyramids as described in Shubnikov (1975) (Fig. 2(b)).

In the majority of cases solid state reactions start at a surface and proceed into the bulk. Both (a)

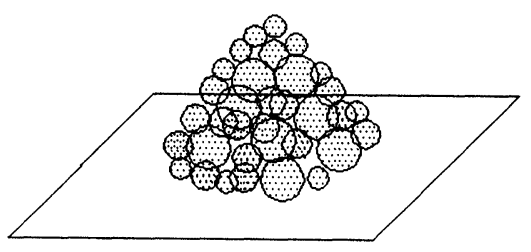

(b)

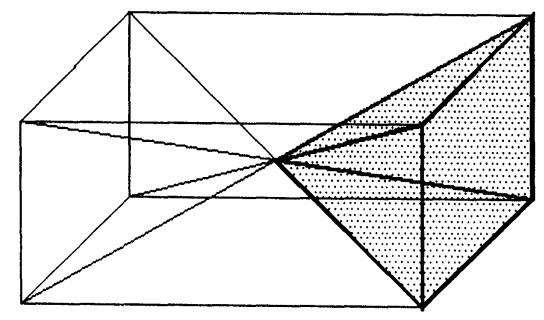

(c)

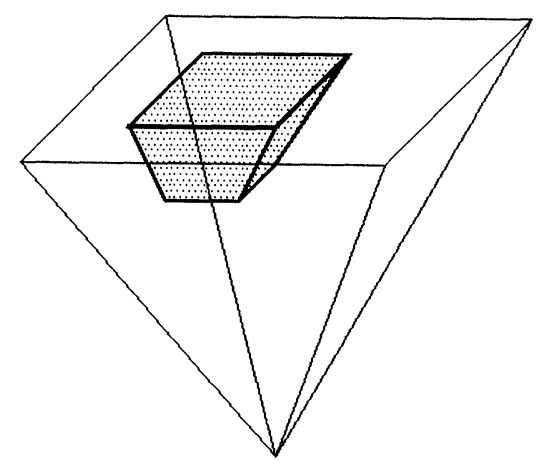

(d)

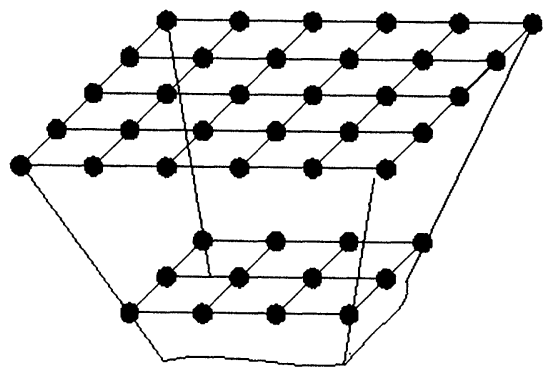

(e)

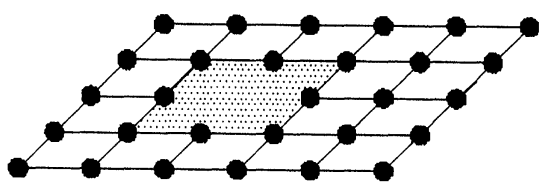

FIGURE 2 The main steps in passing from a polycrystalline samples to the single crystal face as a chemical individual. Explanations are given in the text.

mechanism and kinetics of interactions between the same reagents may be different at different crystal faces. Quite a number of examples may be found in Prodan (1990). Therefore, it is appropriate 
to consider each crystal face with its growth pyramid separately (Fig. 2(c)).

The next point concerns interrelations between symmetry of causes and symmetry of effects, the general principles having been formulated by Curie. When solid state reaction kinetics, e.g. thermal decomposition kinetics, is simulated within the conventional approach by the widely used Avrami-Erofeev equation or some other geometric-probabilistic equation in terms of the degree of conversion $\alpha$, the routine is to prescribe the spherical form to growing nuclei. This is done mainly for formal reasons and, obviously, is in poor agreement with crystal chemical considerations. According to Prodan (1990) the correspondence between localization forms and crystal symmetry is observed, as a rule, provided that the original crystal is carefully prepared and a reaction is carried out under conditions required for the kinetic regime.

The question about form is actually the question what exactly is described. If the description is restricted to the formation and growth of new phase nuclei alone (as is often the case), there is no room for representing the original solid reagent within the formalism. Accordingly, one cannot even talk about representing it as a chemical individual. On the other hand, the formation of a new phase and the demolition of the original crystal phase cannot be described by the same equation except infrequent cases of complete coherence of all steps. In this way we arrive at the necessity to separate the description of the negative crystal growth, the form of a negative crystal being determined by the crystal chemical structure of a solid reagent (in Fig. 2(c) such a negative crystal is hatched).

The next point is the central one in this logical chain: in simulating the negative crystal growth the reaction front advance along the surface and into the bulk must be described separately to take into account the non-equivalence of surface and bulk positions of a crystal structure. In other words, the description must be basically two-dimensional. It is worth noting that this is the direct consequence of symmetry considerations rather than the way to simplify the model representation at the expense of decreasing the dimensionality. There is also a sound formal argument. Reactions start at surfaces and, hence, the two-dimensional nucleation is implied. The geometric-probabilistic approach requires that nucleation and growth must be of the same dimensionality. Two-dimensional nucleation followed by three-dimensional growth would be the violation of the applicability conditions. In more details these and some other arguments were discussed in Korobov (1995b).

It is adequate to represent a growth pyramid as a set of crystallographic planes parallel to the chosen crystal face (Fig. 2(d)). This way of representing the bulk crystal structure is widely used when surfaces and their properties are concerned (see, for example, Ashcroft and Mermin, 1976). The growth of a negative crystal is described separately within each plane (Fig. 2(e)), the bulk process being followed layer-by-layer.

In this way the growth of negative crystals within a separate crystallographic layer is becoming the conceptually central object of formal description.

The next step is determined by the need to represent the growth of a negative crystal in terms of the geometry of the crystal space of a solid reagent. This may be done in terms of planigons, the complete mathematical theory of which is developed in Delone et al. (1978) and Grünbaum and Shephard (1987). In the present context an important advantage of this mathematical tool, in comparison with more habitual crystal lattices, is such a characteristic as the extensional measure. Also, along with symmetry planigons represent the combinatorial-topological structure of the crystal space of a sample. Due to this the description is more detailed: 46 types of planigons correspond to 17 two-dimensional crystallographic groups (Table I). Figure 3(a)-(c) shows three possible planigon structures corresponding to $\mathrm{p} 4$ symmetry group.

There exists one-to-one correspondence between crystal lattices and planigons, and thus in passing from the former to the latter we lose nothing with respect to representing the chemical individuality of a solid reagent. 
TABLE I The number of planigon tessellations corresponding to each two-dimensional crystallographic group

\begin{tabular}{lclccc}
\hline $\begin{array}{l}\text { Symmetry } \\
\text { group }\end{array}$ & $\begin{array}{c}\text { Number of } \\
\text { planigon types }\end{array}$ & $\begin{array}{c}\text { Symmetry } \\
\text { group }\end{array}$ & $\begin{array}{c}\text { Number of } \\
\text { planigon types }\end{array}$ & $\begin{array}{c}\text { Symmetry } \\
\text { group }\end{array}$ & $\begin{array}{c}\text { Number of } \\
\text { planigon types }\end{array}$ \\
\hline p1 & 2 & $\mathrm{pmg}$ & 4 & $\mathrm{p} 3$ & 2 \\
$\mathrm{p} 2$ & 5 & $\mathrm{pgg}$ & 8 & $\mathrm{p} 31 \mathrm{~m}$ & 2 \\
$\mathrm{pm}$ & 1 & $\mathrm{cmm}$ & 2 & $\mathrm{p} 3 \mathrm{~m} 1$ & 1 \\
$\mathrm{pg}$ & 4 & $\mathrm{p} 4$ & 3 & $\mathrm{p} 6$ & 4 \\
$\mathrm{~cm}$ & 3 & $\mathrm{p} 4 \mathrm{~m}$ & 1 & $\mathrm{p} 6 \mathrm{~m}$ & 1 \\
$\mathrm{pmm}$ & 1 & $\mathrm{p} 4 \mathrm{~g}$ & 2 & & \\
\hline
\end{tabular}

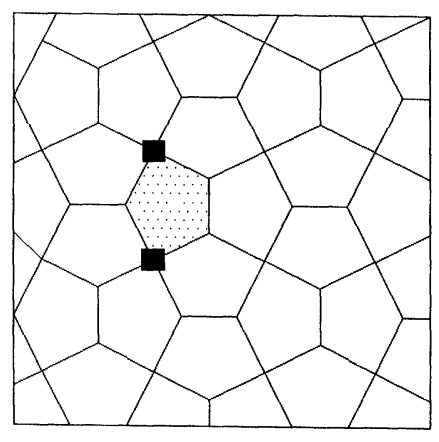

(a)

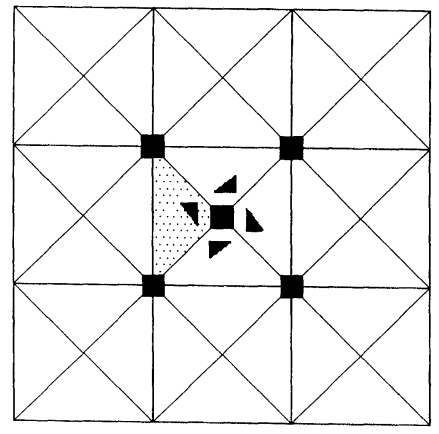

(c)

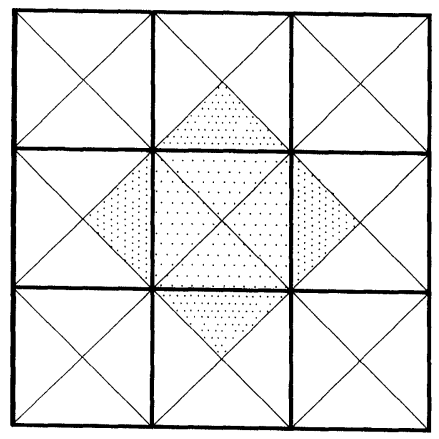

(e)

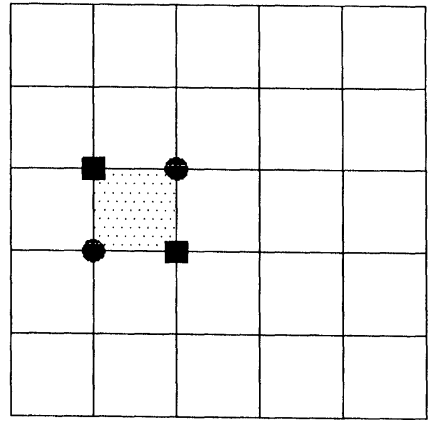

(b)

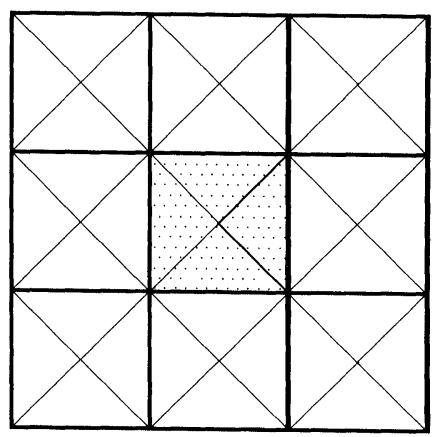

(d)

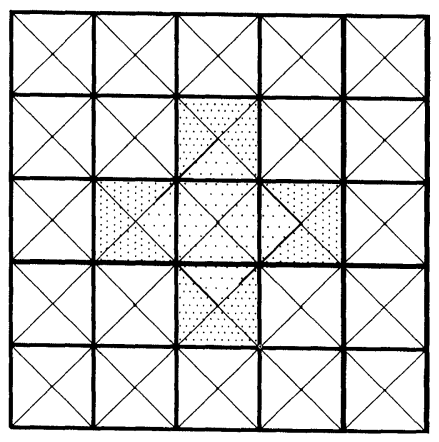

(f)

FIGURE 3 (a)-(c) Three different planigon tessellations for p4 symmetry group, filled quadrangles denote tetrad symmetry axes, filled triangles are asymmetric crystallographic points and show the centers of action of planigons; (d) the superposition of the planigon tessellation (c) and corresponding Wigner-Seitz tessellation; $(e, f)$ first steps of the evolution of a PTCA according to the rule described in the text. 
From the formal viewpoint, a planigon tessellation is a Dirichlet tessellation with respect to lattice points. Each lattice point is the center of action of its planigon. With this in mind, it is reasonable to use another variety of Dirichlet tessellations, WignerSeitz cells, for representing the translational symmetry of a sample (see, for example, Ashcroft and Mermin, 1976). The Wigner-Seitz cell corresponding to the planigon tessellation shown in Fig. 3(c) is hatched by dots in Fig. 3(d). This makes it possible to associate the reaction front advance with the translational symmetry of a sample.

The superposition of a particular planigon tessellation and corresponding Wigner-Seitz tessellation (an example of which is shown in Fig. 3(d)) forms the discrete space which represents full enough the crystal chemical structure of a sample and may be used for developing a discrete basically two-dimensional description of solid state reaction kinetics.

\subsection{Discrete Time: From Geometry to Dynamics}

In the above way the chemical individuality of a solid reagent finds the proper representation in terms of the geometry of the crystal space. To integrate the chemical individuality into dynamic models, they must be agreed with this geometry. The way of doing this is determined by the preferred local occurrence of a solid state reaction. Its chemical essence was clearly interpreted by Langmuir (1916): the very first reaction event promotes substantially the entry of neighbouring atoms into a reaction. With this in mind, the notion of adjacency is used to link geometry of the crystal space and the dynamics of the reaction front advance. (Note that this possibility exists only in two dimensions, see Galiulin and Senchal, 1977.) From this viewpoint one more advantage of Dirichlet tessellations is the possibility to formalize this notion: two points, $p$ and $p_{k}$, of a discrete set of points $\left\{p_{i}\right\}$ are termed adjacent if their Dirichlet domains have a common side.

The significant increase in the probability for the given atom to participate in the elementary single-barrier event provided that one of neigbouring atoms has entered a reaction is represented as a propagation (or transmission) of a reaction. In this context discrete time may be introduced in two ways: (i) assuming the equiprobability of propagation from a given planigon to all adjacent symmetrically equivalent planigons, and (ii) assuming the equiprobability of propagation from a given Wigner-Seitz cell to all adjacent Wigner-Seitz cells. The latter is a bit more rough (more largescaled) variant of the former.

Corresponding to this are two ways of formalizing the unrestricted growth of a separate negative crystal: in terms of cellular automata and in terms of concentric belts.

\subsection{Discrete Dynamics of Unrestricted Growth: Distributed Description in terms of Cellular Automata}

The above superposition of the tessellations may be considered as a cellular automaton (CA), each cell of which consists of planigons (as is shown, for example, in Fig. 3(d)). (Some simple examples of tessellation cellular automata are discussed in Toffoli and Margolus, 1987.) Such CA will be referred to as planigon tessellation cellular automata (PTCA). The only exception is the p1 symmetry group, which serves in our context as a link with conventional CA. It is represented by two tessellations, hexagonal and rectangular, each cell of which is the planigon and Wigner-Seitz cell simultaneously.

In terms of $\mathrm{CA}$, the local interactions are represented as a set of rules according to which a unit or a group of units (a planigon or a cell consisted of planigons) may enter an evolving pattern. First of all we will be interested in the rules that take into account and make explicit the distinctions between translational and non-translational (point) symmetry of a crystal lattice with respect to the reaction front advance.

Consider the planigon tessellations for $\mathrm{p} 4$ symmetry group shown in Fig. 3(c). Planigons in this case are isosceles right triangles (Delone et al., 
1978). All vertexes of the tessellation bear tetrad axes but fall into two types depending on whether four or eight planigons meet at the given vertex. One of the simplest possible rules may be formulated, for instance, as follows:

- Two values, $p=0$ and $p=1$, are possible for a planigon depending on whether it (or, more accurately, an atom situated in its center of action) has entered a reaction. Planigons with $p=1$ are shown by hatching.

- Only two values, 0 and 1, are admitted as well for a square cell.

- The value $c$ of a cell is determined by the values $p$ of component planigons, e.g.

$$
c=p_{1} \oplus p_{2} \oplus p_{3} \oplus p_{4}
$$

where $\oplus$ denotes addition modulo 4 .

- If a planigon or a cell has taken on the value 1, no further changes are possible for it (monotony condition).

- If a cell with the value 0 has in its von Neumann neighbourhood specified as a set of displacement vectors in the form

$$
N_{5}=\left\{\left(\begin{array}{l}
0 \\
0
\end{array}\right) ;\left(\begin{array}{l}
1 \\
0
\end{array}\right) ;\left(\begin{array}{l}
0 \\
1
\end{array}\right) ;\left(\begin{array}{c}
-1 \\
0
\end{array}\right) ;\left(\begin{array}{c}
0 \\
-1
\end{array}\right)\right\},
$$

at least one cell with the value 1 , the reaction front is advanced towards the boundary planigon (Fig. 3(e)).

Note that this rule "keeps" the reaction front inside the cell until all planigons of this cell have entered a reaction. As a result, we get two alternating forms of the evolving pattern (Fig. 3(e) and (f)).

At first glance, the analogy between a regular two-dimensional crystal lattice and a regular lattice of sites of a conventional CA seems to be not only obvious but also trivial. But in describing solidphase chemical reactions in terms of CA, the correspondence between them is not direct. The cells of the PTCA introduced are Wigner-Seitz cells whereas a particular atom of the given sort is represented in these terms by a planigon, in the center of action of which it is situated. Thus, a PTCA cell includes several translationally nonequivalent lattice atoms. This interplay between PTCA cells and planigons provides the possibility to follow the way in which local chemical interactions, describable in terms of planigons, are transformed into involved symmetrical patterns evolving with time in a complicated manner. Due to this PTCA offer the broad possibilities for formulating new rules that are adequate to the wide variety of the experimentally observed localization forms.

\subsection{Discrete Dynamics of Unrestricted Growth: Concentrated Description in terms of Concentric Belts}

Within the approach suggested the advance of the reaction front is associated with the Wigner-Seitz tessellation representing the translational symmetry of a sample. And the simplest discrete description of the unrestricted growth is the description in terms of Wigner-Seitz cells alone. This means that the step of discrete time is taken to be a slightly more large-scaled in comparison with cellular automata. Though not so detailed, the unrestricted growth in terms of Wigner-Seitz cells is analytically tractable. This is material with the account that for the present we have not developed enough analytical tools even for simple one-dimensional cellular automata, as noted in Feldberg et al. (1994).

Two-dimensional Wigner-Seitz cells may be either hexagons or rectangles providing two kinds of patterns (Fig. 4). At each step $s$ (standing in this case for the discrete time) one concentric belt is added to the growing figure (nucleus).

We are interested in the number of cells $C(s)$ that are added at each step $s$. To find it, consider the growing nucleus as a planar graph. Denote the number of faces, edges and vertexes at step $s$ as $F(s), E(s)$ and $V(s)$ respectively. The Euler characteristic

$$
\chi=V-E+F
$$




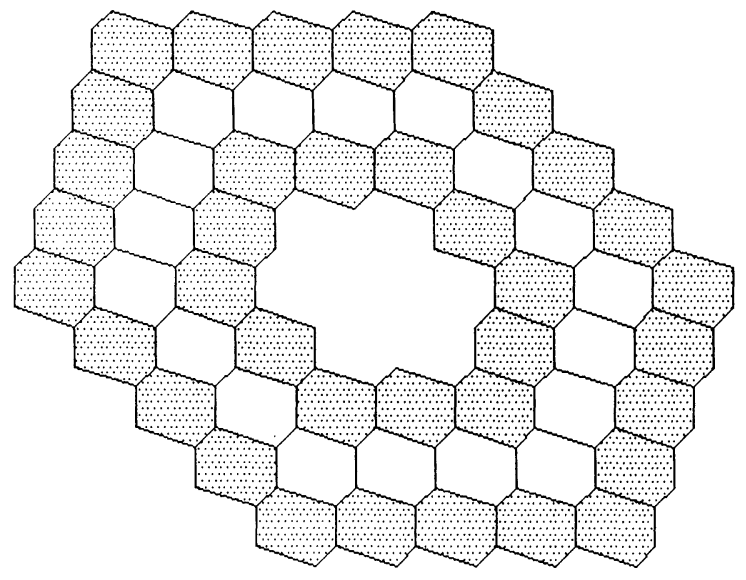

(a)

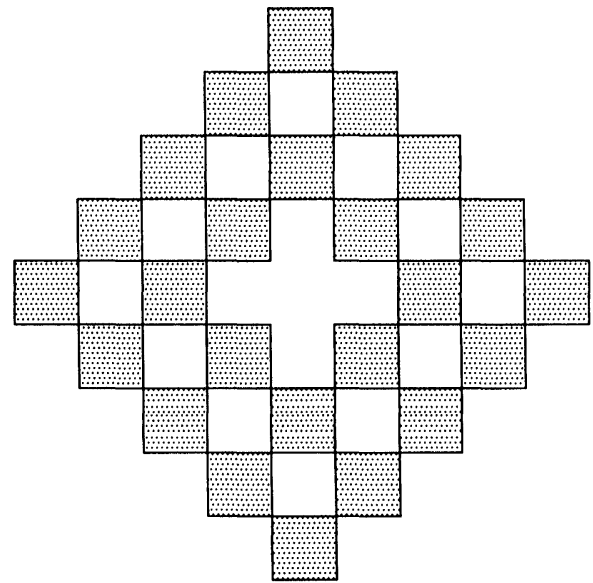

(b)

FIGURE 4 Two possible types of concentric belts; inner and outer belts are shown by hatching.

is equal in this case to unity provided that the outer face is not taken into account (Ore, 1962). In these terms

$$
\begin{aligned}
C(s) & =F(s)-F(s-1) \\
& =[E(s)-E(s-1)]-[V(s)-V(s-1)] .
\end{aligned}
$$

It may be shown (see Korobov, 1996a) that for a separate belt

$$
\begin{aligned}
& E_{\mathrm{b}}(s+1)=\left(\frac{\nu}{2}-1\right) C(s+1)-\frac{\nu}{2}, \\
& E_{\mathrm{B}}(s-1)=\left(\frac{\nu}{2}-1\right) C(s-1)+\frac{\nu}{2},
\end{aligned}
$$

and

$$
E_{\mathrm{B}}-E_{\mathrm{b}}=2 \nu
$$

where indexes $\mathrm{B}$ and $\mathrm{b}$ refer to the external and internal boundaries respectively (see Fig. 4), and $\nu$ is the number of edges of a cell (i.e. either six or four in the present context). Using the known relationship (Ore, 1962) for the number of edges of a graph

$$
2 E=\nu F+E_{\mathrm{B}}
$$

one arrives at the second-order difference equation for $C$

$$
2 C(s)-C(s+1)-C(s-1)=0
$$

which may also be represented in the "one-step" form

$$
\boldsymbol{u}_{s}=M^{s} \boldsymbol{u}_{0}
$$

where $\boldsymbol{u}_{s}=(C(s), C(s+1))$ and

$$
M=\left(\begin{array}{cc}
0 & 1 \\
-1 & 2
\end{array}\right) .
$$

Among various features of this description the following three seem to be the most relevant to the discussed points.

- The reaction front advance is associated with the translational symmetry of the crystal structure in terms of difference equations. This is favourable with the account that the conventional formalism is formulated in terms of differential equations.

- In these terms the growth rate of a separate nucleus is linear. This result is obtained without any special assumptions and is in agreement with 
much experimental evidence (see Barret, 1973; Brown et al., 1980; Delmon, 1969 and references therein).

- This description of the unrestricted growth is purely deterministic.

Thus, in terms of Wigner-Seitz cells it is possible to separate linear deterministic part within essentially nonlinear stochastic problem.

At this point we face an interesting interrelation between deterministic and stochastic description. Real phenomena and processes generally possess both deterministic and stochastic features manifesting them in a larger or smaller measure. In mathematical description of a phenomenon, the routine is to choose either deterministic or stochastic approach depending on the angle it is studied from. In our context we face somewhat different situation: a deterministic part may be singled out within stochastic description, that enables one to get a "chemical insight" into above geometrical universality. As is known, the organization of indeterministic elements into a system may generally result in either enhancement or suppression of this indeterminism (Blechman et al., 1983). The latter is the case when the surface atoms participating in the naturally stochastic single-barrier events are organized into a crystal structure. Due to this, the inherent chemical regularities of a solid state reaction may be described in deterministic terms.

This determines, in its turn, the framework for constructing stochastic part of the description.

\subsection{Discrete Dynamics of Restricted Growth in terms of Random Mosaics}

In reality, the growth of any nucleus is restricted by impingements with neighbouring nuclei. Stochastic part of the problem is represented by two interconnected phenomena: nucleation and nuclei impingements.

One of the main difficulties in describing a solid state reaction is that the above deterministic regularities of the reaction front advance and stochastic regularities of nuclei impingements belong to different levels of the "micro-macro" scale. The meaning of the terms micro and macro is conventional and depends on a particular context. In the present context the macroscopic level is represented by the universal geometrical regularities of firstorder phase transitions. Whereas phase transitions may occur without chemical transformations, any solid state chemical reaction involves disappearance and formation of phases proceeding through nucleation processes. Accordingly, the above geometry representing the chemical individuality of a solid reagent must be also agreed with the geometry of nucleation and nuclei impingements.

The latter may be described in terms of one more variety of Dirichlet tessellations, random mosaics (Møller, 1994; Okabe et al., 1992; Stoyan et al., 1978). In representing various aspects of a solid state reaction in terms of Dirichlet tessellations one makes use of the fact that three varieties of this mathematical notion, planigons, Wigner-Seitz cells, and random mosaics, represent different levels of the micro-macro scale.

Figure 5(a) shows an example of the random mosaic. In the present context it may be interpreted as the ultimate picture after the transformation is completely finished. Thus, carefully prepared metallographic lapping represents such a picture. But another interpretation is also possible. Each cell of a random mosaic may be considered as a "rightful domain" of the seed situated in its center of action. In other words, a seed appearing at some arbitrary time $t$ will assume the form of corresponding cell at $t \rightarrow \infty$. Among various types of random mosaics we will be interested in Voronoi tessellations and Johnson-Mehl tessellations (see Møller, 1994; 1995; Okabe et al., 1992; Stoyan et al., 1978, and references therein). In both cases the Poisson nucleation is assumed. Voronoi case corresponds to the situation when all nuclei appear at once at $t=0$.

Edges of cells of a random mosaic are lines along which neighbouring nuclei impinge, as is sketched in Fig. 5(b). This makes it possible to simulate nuclei impingements as the impingements of a nucleus with the boundary of its cell. One may 


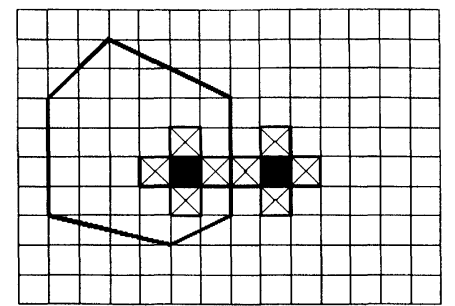

(b)

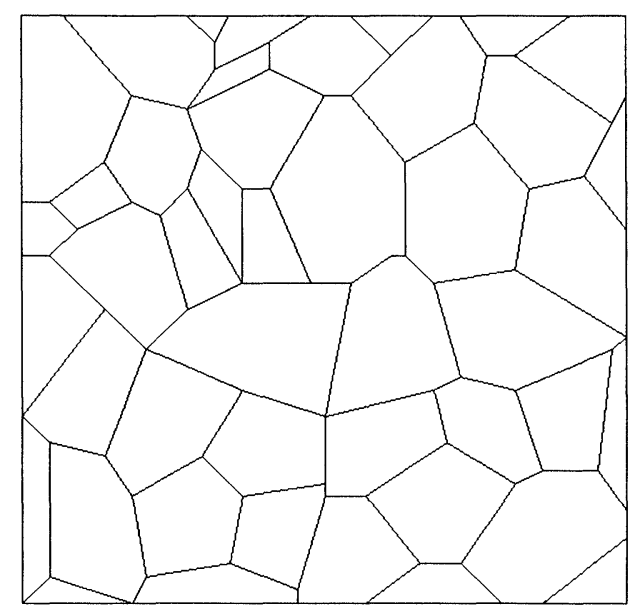

(a)
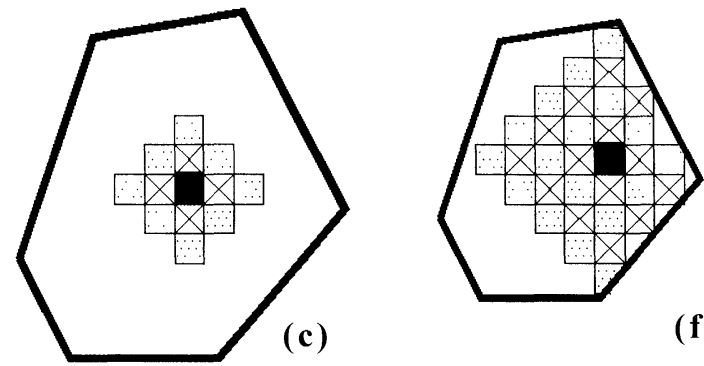

(f)
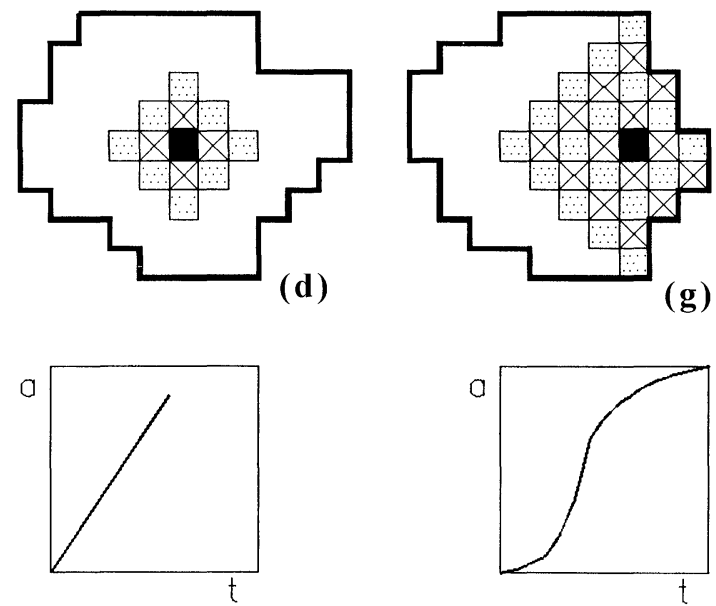

(e)

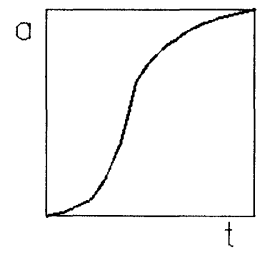

(h)

FIGURE 5 Genesis of "conversion-time" curve in terms of random mosaics. Explanations are given in the text.

define the "local" geometrical degree of conversion as the ratio of the area of the nucleus at time $t$ to the area of corresponding cell and plot the "individual kinetic curve" $\alpha(t)$ for each cell. The omnipresent sigmoid curve "degree of conversion - time", observed experimentally, may be considered as the average over all cells of random mosaic.

This gives an idea about the way in which deterministic and stochastic parts of the problem representing different levels of micro-macro scale may be conjugated. The random mosaic is characterized completely enough by its typical cell (Møller, 1994; Okabe et al., 1992; Stoyan et al., 1978). The typical cell is always a hexagon for which the averaged area, averaged perimeter, and a number of other similar characteristics may be calculated analytically or estimated numerically. Figure 5(c) sketches a typical hexagon with the growing nucleus inside it. The "individual kinetic curve" for a nucleus, which is the center of action of the typical hexagon, will represent the averaged picture.

Straight edges of the typical hexagon generally correspond to the spherical form of nuclei. In contrary to this, nuclei consisted from WignerSeitz cells impinge along stepwise lines the features of which were discussed in Korobov (1995a). To take this into account, one has to construct the typical hexagon in the proper metric (Fig. 5(d)). Until the first impingement with the boundary the growth is linear (Fig. 5(e)) and, therefore, is described by the difference equation (11) for unrestricted growth. In Korobov (1995a) it has been shown that for the growing nucleus restricted by one or two edges of a typical hexagon the linearity is preserved but the slope is different and is determined by the mutual situation of the growing 
nucleus and restricting edges. This means that Eq. (11) holds but $\boldsymbol{u}_{0}$ varies.

In the Voronoi case the random mosaic is time invariant. This is not so for Johnson-Mehl random mosaic, which represents the general case in the present context. The typical hexagon is decreasing all the time due to the appearance of new nuclei in the course of a process. The following visual picture corresponds to this: ever-decreasing averaged hexagonal cell with ever growing nucleus consisted of Wigner-Seitz cells inside it (Fig. 5(f) and (g)). The latter represents the linear deterministic part of the description suggested whereas the former corresponds to its stochastic part. Their interconnection in both time and space are taken into account by their impingements. Six impingements with the edges and six impingements with the angles of an averaged cell provide 12 discontinuity points on the sigmoid $\alpha(s)$ curve, which may be described by 12 conjugated difference equations similar to (11):

$$
\boldsymbol{u}_{\sigma}^{(j)}=M^{\sigma} \boldsymbol{u}_{0}^{(j)}
$$

where $j$ is the consecutive number of a curve segment. The value $j=1$ corresponds to the unrestricted growth. In this case $C(0)=0$ and $C(1)$ is equal to the number of planigon edges. The step

\section{Continual description}

1. The main variable has the geometric-probabilistic meaning alone

2. Applies to new phases formation

3. The chemical individuality of a solid reagent is represented by scalar model coefficients which is insufficient for essentially nonlinear kinetic models

4. The routine is to represent the nucleation as a purely temporal point process

5. Geometrical details of nuclei impingements are taken into account implicitly. This ensures a relative simplicity in obtaining the final results. But in this way the deterministic part of the description is completely "dissolved" in the stochastic part, and the continual description as a whole becomes purely stochastic number $\sigma$ is counted for each segment $j$ off the point next to the corresponding discontinuity point $S_{\mathrm{d}}^{(j-1)}: \sigma=S^{(j)}-S_{\mathrm{d}}^{(j-1)}+1$, i.e. $\sigma=0$ for the first point of each segment. This discrete model corresponds to the continual expression (2) for the rate.

In these terms the genesis of the always sigmoid curve "conversion-time" may be followed and due to this more information may be extracted in comparison with the continual approach in the framework of which geometrical details of impingements are avoided.

\section{CONCLUSIONS}

The main stimulus for developing the discrete alternative to the conventional continual description was to overcome the "chemical facelessness" and to escape in this way the vicious circle of discrimination issues. Though fairly dissimilar, continual and discrete descriptions discussed above have an essential common feature: in both cases we remain within the "nucleation - growth - impingement" conception, and in mathematical respect both descriptions are geometric-probabilistic. With this in mind, the main conceptual aspects of the two approaches may be compared as follows.

\section{Discrete description}

The main variable admits both chemical and geometric-probabilistic interpretations Applies to the original solid reagent

The chemical individuality of a solid reagent is represented in terms of Dirichlet tessellations with due account of the crystal chemical structure.

Nucleation acquires a spatial representation in terms of random mosaics, which is more adequate to the spatial description of nucleus growth.

In the framework of the discrete approach a similar "dissolution" would mean the loss of chemical information. Accordingly, the account of nuclei impingements is explicit: they are represented as the impingements of a separate nucleus with the boundary of the typical cell of corresponding random mosaic 
It may be concluded that the two approaches are fairly disparate in the interpretational capability as well as in the computational efficiency and have good grounds to complement each other in the detailed close description of involved solid state reactions. But for making the simulation as meaningful as possible the first violin in their combination must be played by the discrete description applied to the original solid reagent being represented as a chemical individual.

The other coin of penetration of the ideas and methods of discrete dynamics into the field of heterogeneous chemical kinetics is the possibility to throw a bridge between the islands of homogeneous and heterogeneous kinetics. In passing from homogeneous to heterogeneous kinetic systems we face considerable increase of physicochemical complexity. To answer the question what increase in algoritmic complexity corresponds to this would be interesting in the broad context of developing discrete dynamical models and refining discrete calculus. It seems that suggested approach in terms of Dirichlet tessellations provides some opportunities in this respect.

\section{Acknowledgments}

This work was undertaken at the Chemical Department of Kharkov University. I would like to thank Prof V.D. Orlov and Dr. Yu.V. Kholin for their encouragement.

\section{References}

Ashcroft, N.W. and Mermin, N.D. (1976). Solid State Physics. Holt, Rinehart and Winston, New York.

Avrami, M. (1941). Kinetics of phase changes III. J. Phys. Chem. 9, $177-184$.

Barret, P. (1973). Cinétique Hétérogène. Gauthier-Villars, Paris. Belen'kiy, V.Z. (1980). Geometric - Probabilistic Models of Crystallization. Nauka, Moscow (in Russian).

Blechman, I.I., Myshkis, A.D. and Panovko, J.G. (1983). Mechanics and Applied Mathematics: Logic and Peculiarities of Mathematical Description. Nauka, Moscow (in Russian).

Bradley, M.R. (1987). Comment on "Ring Dynamics and Percolation in an Excitable Medium". J. Chem. Phys. 86, $7242-7246$.
Brown, M.E., Dollimore, D. and Galwey, A.K. (1980). Reactions in the Solid State. Elsevier, Amsterdam.

Careri, G. (1982). Ordine e Disordine Nella Materia. Laterza, Roma Bari.

Chiu, S.N. (1995). Limit theorems for the time of completion of Johnson-Mehl tessellations. Adv. Appl. Prob. 27, 889-910.

Delmon, B. (1969). Introduction à la Cinétique Hétérogène. Éditions Technip, Paris.

Delone, B.N., Dolbilin, N.P. and Shtogrin, M.P. (1978). Combinatorial and metrical theory of planigons. Proc. Math. Inst. Acad. Sci. USSR 148, 109-140 (in Russian).

Feldberg, R. (1994). Recursive definition of global cellularautomata mappings. Phys. Rev. E. 49, 1699-1711.

Feynman, R. (1965). The Character of Physical Law. Cox and Wyman LTD, London.

Galiulin, R.V. and Senchal, M. (1977). About some recent progress and issues of the symmetry theory, in Patterns of Symmetry (Senechal, $\mathrm{M}$ and Fleck, G., Eds.), Univ. of Massachusetts Press, Amherst.

Haken, H. (1983). Advanced Synergetics. Springer-Verlag, Berlin.

Johanson, W.A. and Mehl, R.F. (1939). Reaction kinetics in processes of nucleation and growth. Trans. Amer. Inst. Ming. Metal. Engrs. 135, 416-458.

Kallenberg, O. (1983). Random Measures. Akademie Verlag, Berlin.

Kapral, R. (1991). Discrete models for chemically reacting systems. J. Math. Chem. 6, 113-163.

Kiperman, S.L. (1979). Foundations of Chemical Kinetics in Heterogeneous Catalysis. Chemistry, Moscow (in Russian).

Kolmogorov, A.N. (1937). On statistical theory of metal crystallization. Izvestia Akademy of Science SSSR, Ser. Math. 3, 355-358 (in Russian).

Korobov, A. (1993a). The rate of a heterogeneous chemical reaction as a measure of the random marked-point process. Thermochim. Acta 224, 281-289.

Korobov, A. (1993b). Solid-phase reaction kinetics: meaningful simulation versus formal approximation. J. Thermal Anal. 39, $1451-1458$.

Korobov, A. (1994). A geometric-probabilistic approach to heterogeneous chemical kinetics with respect to the IKP ambiguity. Thermochim. Acta 243, 79-93.

Korobov, A. (1995a). Heterogeneous chemical kinetics: a twodimensional insight into sigmoid $\alpha-t$ curve. J. Math. Chem. 17, 323-333.

Korobov, A. (1995b). Localization phenomena in heterogeneous chemical kinetics with respect to a geometric-probabilistic description. Thermochim. Acta 254, 1-10.

Korobov, A. (1996a). Heterogeneous chemical kinetics in two dimensions: two ways of discrete description. Thermochim. Acta 279, 191-204.

Korobov, A. (1996b). Solid-phase reaction kinetics: towards deeper insight through a discrete description. Heterogeneous Chemistry Reviews 3, 477-497.

Langmuir, I. (1916). The constitution and fundamental properties of solids and liquids. J. Amer. Chem. Soc. 38, 2221-2295.

Møller, J. (1992). Random Johnson-Mehl Tessellations. Adv. Appl. Prob. 24, 814-844.

Møller, J. (1994). Lectures on Random Voronoi Tessellations. Lecture notes in Statistics Vol. 87, Springer-Verlag, New York.

Møller, J. (1995). Generation of Johnson-Mehl crystals and comparative analysis of models for random nucleation. $A d v$. Appl. Prob. 27, 367-383.

Nicolis, G. and Prigogine, I. (1989). Exploring Complexity. W.H. Freeman and Co., New York. 
Okabe, A., Boots, B.N. and Sugihara, K. (1992). Spatial Tessellations, Concepts and Applications of Voronoi Diagrams. Wiley, New York.

Ore, O. (1962). Theory of Graphs. AMS, Providence.

Packard, N.H. and Wolfram, S. (1985). Two-dimensional cellular automata. J. Stat. Phys. 38, 901-919.

Prodan, E.A. (1990). Topochemistry of Crystals. Nauka and Technika, Minsk (in Russian).

Šestak, J. (1984). Thermophysical Properties of Solids, their Measurements and Theoretical Thermal Analysis. Elsevier, Amsterdam.
Shubnikov, A.V. (1975). Selected Papers on Crystallography. Nauka, Moscow (in Russian).

Stoyan, D., Kendall, W.S. and Mecke, J. (1978). Stochastic Geometry and its Applications. Wiley, Chichester.

Toffoli, T. and Margolus, N. (1987). Cellular Automata Machines. MIT Press, Cambridge, MA.

Weinberg, M. and Kapral, R. (1989). Phase transition kinetics in finite inhomogeneously nucleated systems. J. Phys. Chem. 91, 7146-7152. 


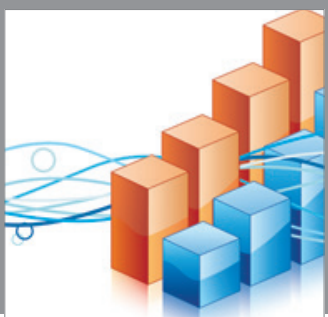

Advances in

Operations Research

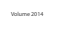

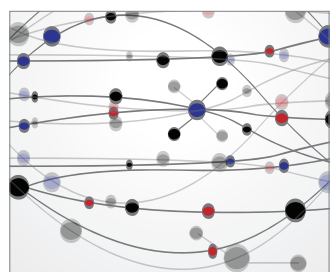

\section{The Scientific} World Journal
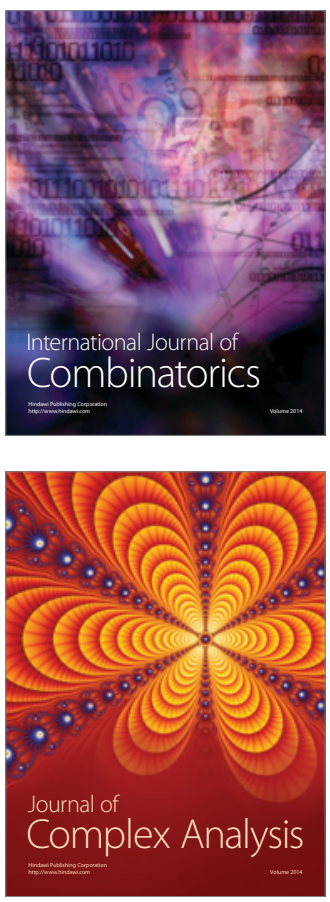

International Journal of

Mathematics and

Mathematical

Sciences
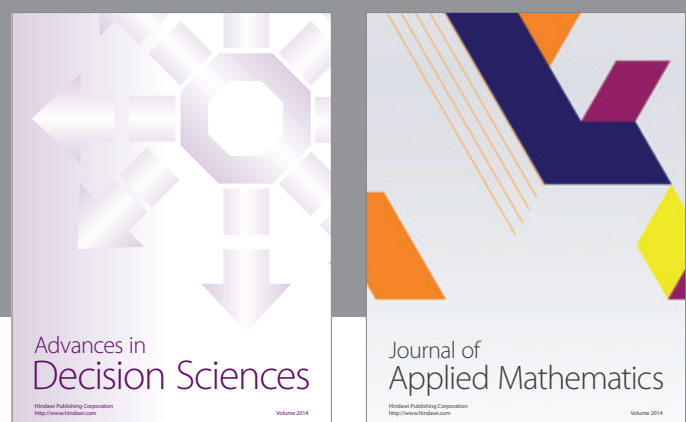

Journal of

Applied Mathematics
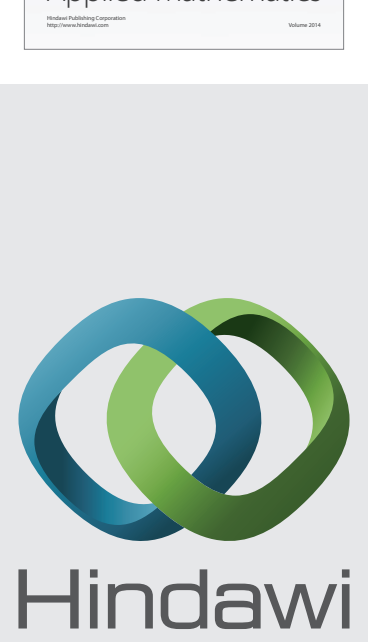

Submit your manuscripts at http://www.hindawi.com
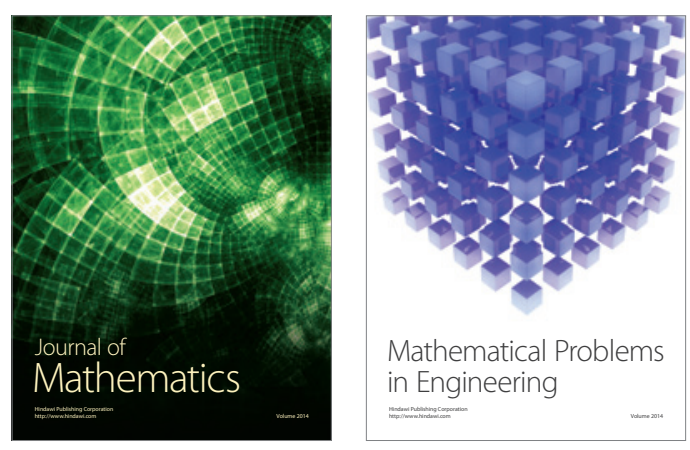

Mathematical Problems in Engineering
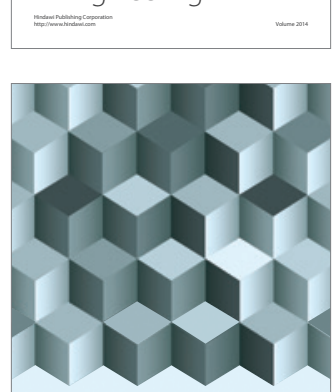

Journal of

Function Spaces
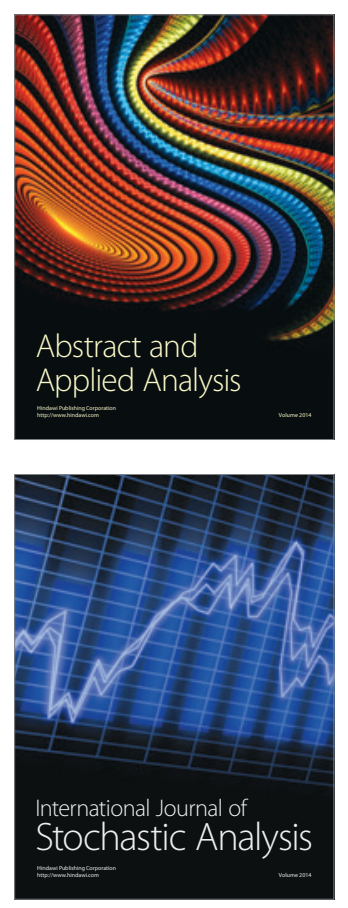

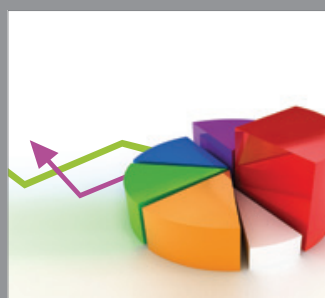

ournal of

Probability and Statistics

Promensencen
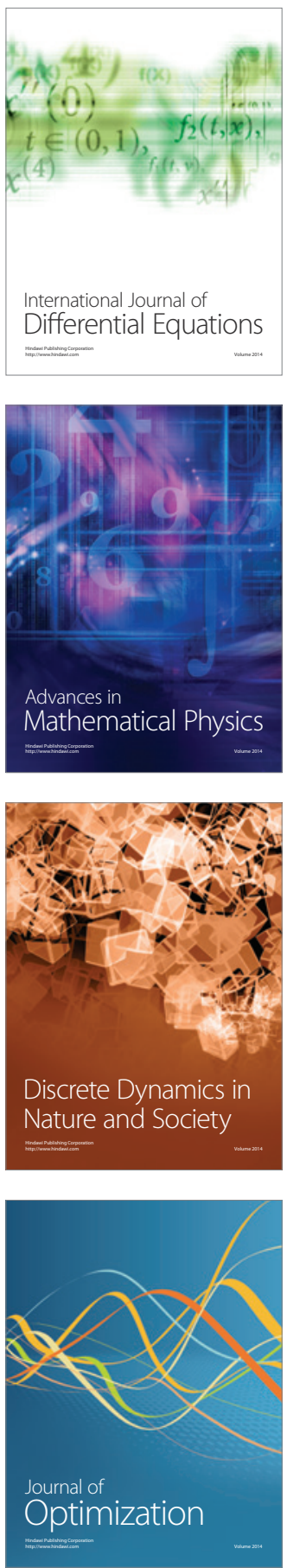